\title{
Systemic lupus erythematosus with intestinal perforation: A case report
}

\author{
YUQING GU $^{1}$, TAO ZHU ${ }^{2}$, YIQING WANG ${ }^{1}$ and HONGXING XU ${ }^{1}$ \\ Departments of ${ }^{1}$ General Surgery, and ${ }^{2}$ Intensive Care Unit, Taicang Hospital Affiliated to Soochow University, \\ Taicang, Jiangsu 215400, P.R. China
}

Received September 9, 2014; Accepted July 7, 2015

DOI: $10.3892 /$ etm.2015.2639

\begin{abstract}
Systemic lupus erythematosus (SLE) is a systemic autoimmune inflammatory disease, which can affect almost all systems and organs. Gastrointestinal disorder is one of the most noteworthy complications of patients with SLE. However, gastrointestinal disorder with intestinal perforation is rare, but potentially life-threatening if not treated promptly. The present study reported a case of SLE with intestinal perforation, where surgical intervention was performed and a crevasse $(\sim 3 \mathrm{~cm}$ in diameter) was detected in the ileum, $60 \mathrm{~cm}$ from the ileocecal valve. Following surgery, the patient suffered from difficult ventilator weaning, septic shock and intestinal obstruction. The patient was successfully treated and discharged from the hospital after $\sim 4$ months of treatment. Intestinal perforation in SLE patients is potentially life-threatening; early diagnosis and prompt treatment are crucial to the management of this rare complication of SLE.
\end{abstract}

\section{Introduction}

Systemic lupus erythematosus (SLE) is an autoimmune disease with multi-systemic clinical symptoms. The regions that are most affected by SLE are the skin and the musculoskeletal, hematopoietic and cardiopulmonary systems (1). Digestive disorders are less common, but potentially much more serious, and perforation of the intestinal tract is one of the most severe complications of SLE. SLE is caused by gastrointestinal vasculitis and thrombosis, and is considered to be life-threatening $(2,3)$. The clinical symptoms of lupus enteritis are nonspecific, but generally include abdominal pain, vomiting, diarrhea and fever. Typical computed tomography (CT) features of SLE include bowel wall edema, mesenteric abnormalities and ascites. Lupus enteritis is steroid responsive

Correspondence to: Dr Hongxing Xu, Department of General Surgery, Taicang Hospital Affiliated to Soochow University, 58 South Changsheng Road, Taicang, Jiangsu 215400, P.R. China E-mail: xuhongxing61@163.com

Key words: systemic lupus erythematosus, intestinal perforation and typically has an excellent overall prognosis. If not treated correctly, SLE may develop to intestinal necrosis and perforation of the intestinal tract, which is among the most severe complications of SLE $(4,5)$. The present study reported a case of SLE with intestinal perforation, which required surgical intervention. The patient was successfully treated.

\section{Case report}

A 44-year-old female patient was admitted to the Taicang Hospital Affiliated to Soochow University (Taicang, China) in November 9, 2013, presenting with abdominal pain and bloating, nausea and vomiting for $24 \mathrm{~h}$, as well as no bowel movements for 2 days. The patient had been diagnosed with systemic lupus erythematosus (SLE) and lupus nephritis (LN) 16 years prior to admission, and received steroid therapy (prednisone, $10 \mathrm{mg} /$ day). Written informed consent was obtained from the patient. In November 2010, April 2011 and June 2013, the patient had been hospitalized in the Department of Gastroenterology of the Taicang Hospital for chronic intestinal pseudo-obstruction (CIP). The patient had been successfully treated using nonsurgical treatment, which included fasting, gastrointestinal decompression, gastromotor drug administration and parenteral nutrition. In addition, the patient had a history of high blood pressure for 3 years and received amlodipine at $5 \mathrm{mg} /$ day for 3 years.

In November 9, 2013, the patient was again admitted to the hospital and treated with conservative therapy; however, $10 \mathrm{~h}$ after admission, the patient complained of gradually increasing abdominal pain. The results of physical examination were within normal ranges, as follows: Temperature, $36.7^{\circ} \mathrm{C}$; blood pressure, $90 / 45 \mathrm{mmHg}$; heart rate, $72 / \mathrm{min}$. Abdomen palpation revealed muscular defense with diffuse tenderness and rebounding pain, particularly in the lower area. No palpable mass was observed and bowel sounds were hypoactive.

The data collected from the laboratory examinations are summarized in Table I. The patient's peripheral blood showed leucopenia, but the percentage of neutrophils was markedly increased. Blood chemistry tests revealed hypoproteinemia and hypoalbuminemia, while liver function test results were normal and renal function tests revealed a slight increase in blood urea nitrogen and marked levels of C-reactive protein. The erythrocyte sedimentation rate was markedly elevated. 
Table I. Laboratory data gathered following admission and prior to discharge.

\begin{tabular}{|c|c|c|}
\hline Tests & $\begin{array}{l}\text { Results at } \\
\text { admission }\end{array}$ & $\begin{array}{l}\text { Results at } \\
\text { discharge }\end{array}$ \\
\hline \multicolumn{3}{|l|}{$\mathrm{CBC}$} \\
\hline WBC $\left(3.7-9.2 \times 10^{9} / 1\right)$ & 3.0 & 4.7 \\
\hline Neutro. $(45-80 \%)$ & 87.7 & 72.2 \\
\hline Lymph. (20-40\%) & 9.3 & 21.4 \\
\hline $\operatorname{RBC}\left(3.7-5.1 \times 10^{12} / 1\right)$ & 4.63 & 3.67 \\
\hline $\mathrm{Hb}(113-151 \mathrm{~g} / \mathrm{l})$ & 126 & 111 \\
\hline Hct (33.5-45.0) & 40.6 & 34.1 \\
\hline Plt $\left(101-320 \times 10^{9} / 1\right)$ & 168 & 257 \\
\hline \multicolumn{3}{|l|}{ Blood chemistry } \\
\hline $\mathrm{TP}(60-83 \mathrm{~g} / 1)$ & 38.0 & 48.6 \\
\hline Alb (35-54 g/l) & 16.1 & 27.3 \\
\hline $\mathrm{Na}(135-145 \mathrm{mmol} / \mathrm{l})$ & 139.3 & 141.0 \\
\hline $\mathrm{K}(3.5-5.2 \mathrm{mmol} / \mathrm{l})$ & 3.7 & 3.9 \\
\hline $\mathrm{Cl}(96-108 \mathrm{mmol} / \mathrm{l})$ & 109.9 & 109.8 \\
\hline BUN (2.9-8.2 mmol/l) & 11.2 & 6.06 \\
\hline $\mathrm{Cr}(40-120 \mathrm{umol} / \mathrm{l})$ & 97.0 & 38.0 \\
\hline T-Bil (2-25 umol/l) & 20.7 & 7.5 \\
\hline AST (0-40 U/1) & 24.0 & 17.2 \\
\hline ALT (0-40 U/1) & 16.0 & 16.1 \\
\hline$\gamma \mathrm{GT}(5-50 \mathrm{U} / \mathrm{l})$ & 14.0 & 154.5 \\
\hline LDH (109-245 U/l) & 117.0 & 165.9 \\
\hline ESR $(0-20 \mathrm{~mm} / \mathrm{H})$ & 100 & 5 \\
\hline CRP (1-10 mg/l) & 172 & 5.9 \\
\hline \multicolumn{3}{|l|}{ Serological test } \\
\hline $\operatorname{IgG}(6.94-16.20 \mathrm{~g} / 1)$ & 3.9 & 12.0 \\
\hline $\operatorname{IgA}(0.68-3.78 \mathrm{~g} / \mathrm{l})$ & 0.72 & 1.57 \\
\hline $\operatorname{IgM}(0.60-2.63 \mathrm{~g} / \mathrm{l})$ & 0.44 & 2.35 \\
\hline C3 $(0.88-2.01 \mathrm{~g} / \mathrm{l})$ & 0.28 & 0.94 \\
\hline $\mathrm{C} 4(0.1-0.4 \mathrm{~g} / \mathrm{l})$ & 0.14 & 0.30 \\
\hline Anti-dsDNA (negative) & $( \pm)$ & $(-)$ \\
\hline Anti-RNP (negative) & $(-)$ & $(-)$ \\
\hline Anti-Sm (negative) & $(-)$ & $(-)$ \\
\hline SSA (negative) & $(-)$ & $(-)$ \\
\hline SSB (negative) & $(+)$ & $(-)$ \\
\hline \multicolumn{3}{|l|}{ Hemostatic date } \\
\hline PT (9.6-14.3s) & 12.9 & 14.0 \\
\hline APTT (23.7-36.4s) & 29.3 & 27.8 \\
\hline AT3 (75.0-130.0\%) & 105.7 & 113.0 \\
\hline $\mathrm{Fgb}(1.7-4.1 \mathrm{~g} / \mathrm{l})$ & 2.60 & 2.52 \\
\hline INT (0.85-1.25) & 1.09 & 1.06 \\
\hline \multicolumn{3}{|l|}{ Tumor marker } \\
\hline $\operatorname{CEA}(0.00-6.00 \mathrm{ng} / \mathrm{ml})$ & 2.55 & Not performed \\
\hline $\operatorname{AFP}(0.00-8.00 \mathrm{ng} / \mathrm{ml})$ & 1.91 & Not performed \\
\hline CA199 (0.00-30.00 U/ml) & 0.6 & Not performed \\
\hline
\end{tabular}

$\mathrm{CBC}$, complete blood count; WBC, white blood cell count; $\mathrm{RBC}$, red blood cell count; $\mathrm{Hb}$, hemoglobin; Hct, hematocrit; Plt, platelet count; TP, total protein; Alb, albumin; BUN, blood urea nitrogen; T-Bil, total bilirubin; ALT, alanine transaminase; AST, aspartate aminotransferase; $\gamma \mathrm{GT}, \gamma$-glutamyl transpeptidase; $\mathrm{LDH}$, lactate dehydrogenase; ESR, erythrocyte sedimentation rate; CRP, C-reactive protein; Ig, immunoglobulin; C, complement component; RNP, ribonucleoprotein; anti-Sm, anti-Smith; SSA, Ro antibodies; SSB, La antibodies; PT, prothrombin time; APTT, activated partial thromboplastin time, AT3, antithrombin III; Fgb, fibrinogen- $\beta$; INT, international normalised ratio; CEA, carcinoembryonic antigen; AFP, $\alpha$-fetoprotein; CA199, carbohydrate antigen 199.
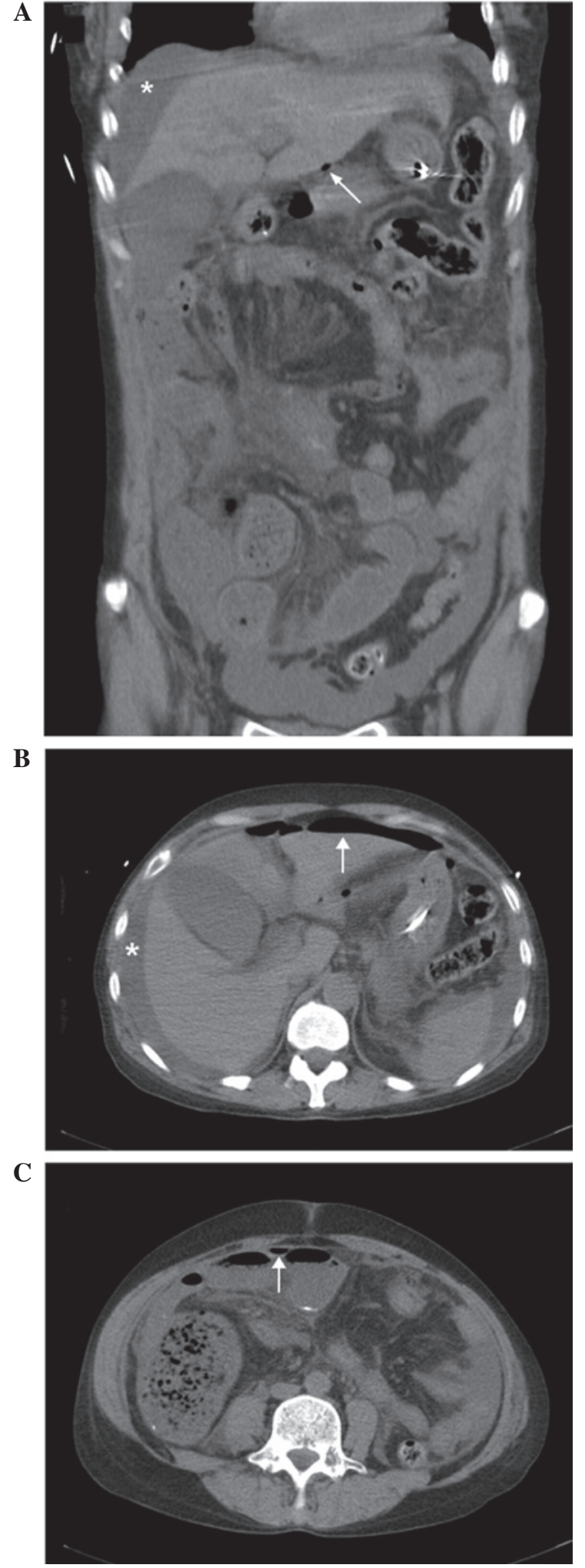

Figure 1. Computer tomography scans of the abdominal cavity of the patient. (A) Coronary, (B) porta hepatis plane and (C) umbilical plane transverse slices of the abdominal computer tomography scan revealing the free fluid (asterisk) and free gas (arrow) in the patient's abdomen.

Immunological tests revealed positive results for anti-SSB antibodies, weakly positive for anti-dsDNA and negative for anti-ribonucleoprotein, anti-Smith and Ro antibodies. In addition, the levels of serum immunoglobulin $(\mathrm{Ig}) \mathrm{G}, \mathrm{IgM}$ and complement component (C)3 were decreased, while those of 


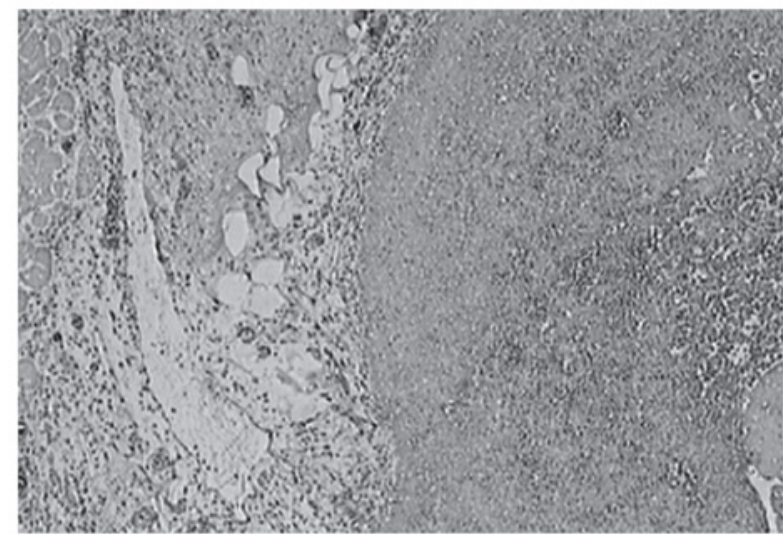

Figure 2. Histological examination of the resected small intestine. Necrosis and perforation of the bowel wall, and inflammatory cell infiltrating into the full-thickness of the bowel wall were observed (magnification, x40; stain, hematoxylin and eosin).

serum IgA and C4 levels were normal. The test results from the coagulo-fibrinolytic system were all normal.

An abdominal CT scan was performed (Fig. 1), which revealed a large volume of free fluid and gas in the abdominal cavity and an area suspicious for intestinal perforation. Diagnostic abdominocentesis was performed and $5 \mathrm{ml}$ muddy straw-colored peritoneal fluid was aspirated without any obstructions.

The patient underwent an emergency exploratory laparotomy and the intraoperative findings confirmed the initial diagnosis of intestinal perforation. Large feces and intestinal contents were observed in the abdominal cavity, as well as severe adhesions between the ileum $(40-80 \mathrm{~cm}$ from the ileocecal valve) and adjacent parietal peritoneum. A crevasse with a diameter of $\sim 3 \mathrm{~cm}$ was detected in the ileum, $\sim 60 \mathrm{~cm}$ from the ileocecal valve. Several other small perforations of the ileum, $\sim 50 \mathrm{~cm}$ from the ileocecal valve, were observed, and the bowel wall in that area was overly thin. The appendix was found to have chronic inflammation, since fecal stone blocked the opening of the cavity. Inspection of the entire abdominal cavity revealed no other evident lesions. An enterolysis, appendectomy and distal ileectomy $(50 \mathrm{~cm}$ of the diseased small bowel) with an ileostomy were performed. Histological examination (Fig. 2) of the resected small bowel tissue revealed necrosis, perforation and inflammatory cell infiltration with bleeding observed on the full-thickness of the bowel wall, while the removed appendix confirmed the diagnosis of chronic appendicitis.

In addition, the patient suffered decannulation failure and difficult weaning from the ventilator. Following four failed attempts of decannulation, tracheotomy was perform on December 2, 2013. Mechanical ventilation support was used discontinuously and the tracheal incision was sealed on January 22, 2014. Continuous anesthesia of midazolam and fentanyl was administered intravenously for sedation and pain control.

The patient also suffered from septic shock and received a cardiac dose of norepinephrine for approximately half a month, until November 26, 2013, and antibiotics were administered according to the bacterial culture and drug sensitivity results. At the end of December 2013, the patient's fever and white blood cell count had alleviated, and the inflammation had decreased. At postoperative day 4, the patient started drinking small volumes of water via a nose-jejunum nutrition tube, and a few days later she was able to consume a liquid diet. During the liquid diet phase, the patient was treated with $10 \mathrm{mg}$ prednisolone daily. On December 04, 2013, the patient developed abdominal pain, nausea and vomiting; subsequently, the dose of prednisolone was increased to $40 \mathrm{mg} /$ day for pulse-dose therapy and the symptoms were relieved. Following the termination of the pulse-dose therapy, the prednisolone dose was decreased and the patient developed intestinal obstruction again; however, the patient's condition improved soon after fasting for 3 days. Right lower extremity deep vein thrombosis was observed on November 20,2013 , and heparin therapy was performed for 2 weeks. The postoperative clinical course of the patient is shown in Fig. 3. Treatment was successful and the patient was discharged on March 3, 2014 and has exhibited no further complications to date.

\section{Discussion}

Systemic lupus erythematosus (SLE) is a systemic autoimmune inflammatory disease that can affect almost every organ and system of the human organism. Gastrointestinal disorder is one of the most noteworthy complications of SLE, since it can be life-threatening if not treated promptly $(6,7)$. The most common SLE-associated gastroenteropathies include protein-losing enteropathy, lupus mesenteric vasculitis, acute pancreatitis, intestinal pseudo-obstruction and other rare complications, such as inflammatory bowel diseases and celiac disease (8).

Chronic intestinal pseudo-obstruction (CIP) is characterized by ineffective intestinal propulsion without any mechanical obstruction of the gut (9). Symptoms of CIP include abdominal pain and distension, nausea and vomiting, constipation and weight loss. Radiographs of the abdomen demonstrate fluid in the bowel loops and a thickened intestinal wall. CIP is considered to be caused by the autoimmune inflammatory involvement of the visceral smooth muscle and enteric nervous system (10). Nonsurgical treatments that are often effective against CIP include high doses of corticosteroids, fasting, nasogastric drainage, total parenteral nutrition and administration of gastromotor or anti-infective drugs (11). In the present case, CIP was diagnosed and, although the patient had previously been treated by conservative therapy, the disease reappeared three more times.

At the fourth time of hospitalization, conservative therapy was no longer effective. The patient complained of gradually increasing abdominal pain, and abdomen palpation revealed muscular defense and diffuse pain with rebound tenderness. The abdominal CT scan revealed a large amount of free fluid and gas in the abdominal cavity and an area suspicious for intestinal perforation. An emergency exploratory laparotomy confirmed the diagnosis of bowel infarction and perforation. Bowel infarction and perforation in patients with SLE are often caused by acute ischemic enteritis of the small intestine, which is also described as lupus mesenteric vasculitis $(12,13)$. Lupus mesenteric vasculitis is an uncommon, but severe complication occurring in patients with SLE. In Asia, the overall 

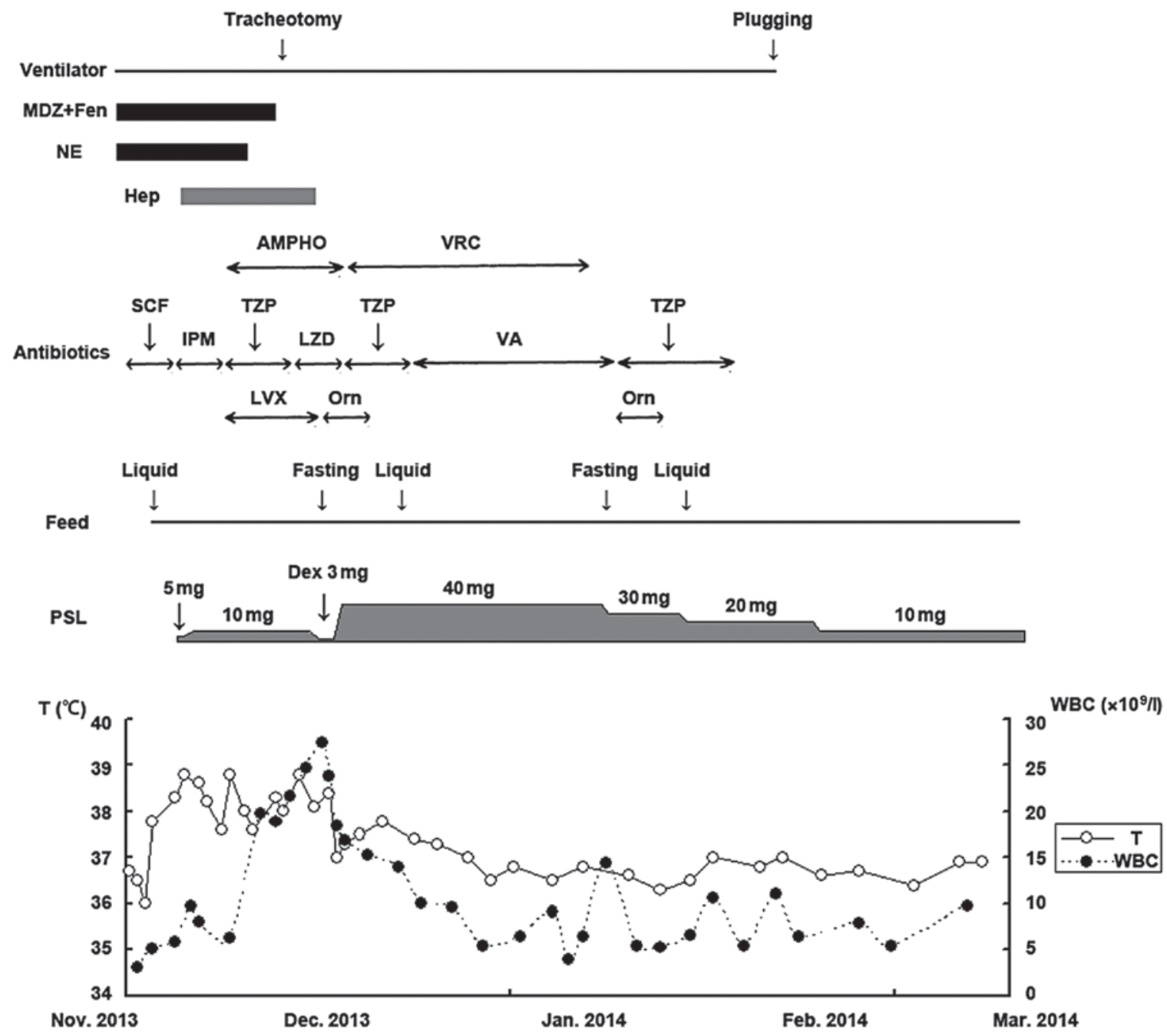

Figure 3. Post-operative clinical course of the patient. MDZ, midazolam; Fen, fentanyl; NE, norepinephrine; Hep, heparin; AMPHO, amphotericin B; VRC, voriconazole; SCF, cefoperazone/sulbactam; IPM, imipenem; TZP, piperacillin/tazobactam; LZD, linezolid; VA, vancomycin; LVX, levofloxacin; Orn, ornidazole; PSL, prednisolone; Dex, dexamethasone; WBC, white blood cell count; T, T cells.

prevalence of lupus mesenteric vasculitis in patients with SLE has been reported to be $2.2-9.7 \%(14,15)$. Lupus mesenteric vasculitis is considered to be caused in patients with SLE by circulating autoantibodies that form an immune complex deposition in blood vessels, which can lead to the development of vasculitis and thrombosis of the vessels supplying the intestine. An inadequate blood supply to the intestine results in ulceration, infarction and perforation $(16,17)$.

The prognosis of SLE patients with intestinal perforation is poor. Therefore, early diagnosis and appropriate interventions are crucial to the management of the disease (18). The majority of SLE cases have been found to be long-term users of corticosteroids and immunosuppressants, which are known to weaken the immune system $(19,20)$. Any surgical intervention is more risky in SLE patients compared with individuals without SLE $(21,22)$. In the present case, the patient faced three major problems following surgery. The patient suffered from decannulation failure and difficult weaning from the ventilator, and thus tracheotomy was performed. In addition, the patient suffered from septic shock for which she received a cardiac dose of norepinephrine for approximately half a month, and antibiotics were administered according to the bacterial culture and drug sensitivity results, until the white blood cell count and temperature returned to normal. Finally, a correlation was observed between prednisolone dose and intestinal obstruction: At postoperative day 25, the patient developed intestinal obstruction; thus, the dose of prednisolone was increased for pulse-dose therapy and the symptoms were relieved. However, at 2 months postoperatively, the dose of prednisolone was gradually reduced and intestinal obstruction reappeared. The patient then received conservative treatment once again, including fasting and parenteral nutrition.

In conclusion, intestinal perforation in SLE patients is extremely rare; however, it can potentially be life-threatening. Early diagnosis and prompt treatment are crucial to the management of this rare complication of SLE.

\section{References}

1. Meszaros ZS, Perl A and Faraone SV: Psychiatric symptoms in systemic lupus erythematosus: A systematic review. J Clin Psychiatry 73: 993-1001, 2012.

2. Janessens P, Arnaud L, Galicier L, Mathian A, Hie M, Sene D, Haroche J, Veyssier-Belot C, Huynh-Charlier I and Grenier PA: Lupus enteritis: From clinical findings to therapeutic management. Orphanet J Rare Dis 8: 67, 2013. 
3. Malaviya AN, Sharma A, Agarwal D, Kapoor S, Garg S, Singh S and Rawat R: Acute abdomen in SLE. Int J Rheum Dis 14 98-104, 2011.

4. Chng HH, Tan BE, Teh CL and Lian TY: Major gastrointestinal manifestations in lupus patients in Asia: Lupus enteritis, intestinal pseudo-obstruction and protein-losing gastroenteropathy. Lupus 19: 1404-1413, 2010.

5. Xu D, Yang H, Lai CC, Li P, Zhang X, Yang XO, Zhang FC and Qian JM: Clinical analysis of systemic lupus erythematosus with gastrointestinal manifestations. Lupus 19: 866-869, 2010.

6. Medina F, Ayala A, Jara LJ, Becerra M, Miranda JM and Fraga A: Acute abdomen in systemic lupus erythematosus: The importance of early laparotomy. Am J Med 103: 100-105, 1997.

7. Al-Hakeem MS and McMillen MA: Evaluation of abdominal pain in systemic lupus erythematosus. Am J Surg 176: 291-294, 1998.

8. Tian XP and Zhang X: Gastrointestinal involvement in systemic lupus erythematosus: Insight into pathogenesis, diagnosis and treatment. World J Gastroenterol 16: 2971-2977, 2010.

9. Mok MY, Wong RW and Lau CS: Intestinal pseudo-obstruction in systemic lupus erythematosus: An uncommon but important clinical manifestation. Lupus 9: 11-18, 2000.

10. Garcia López CA, Laredo-Sánchez F, Malagón-Rangel J, Flores-Padilla MG and Nellen-Hummel H: Intestinal pseudo-obstruction in patients with systemic lupus erythematosus: A real diagnostic challenge. World J Gastroenterol 20: $11443-11450,2014$

11. Khairullah S, Jasmin R, Yahya F, Cheah TE, Ng CT and Sockalingam S: Chronic intestinal pseudo-obstruction: A rare first manifestation of systemic lupus erythematosus. Lupus 22: 957-960, 2013.

12. Ahn E, Luk A, Chetty R and Butany J: Vasculitides of the gastrointestinal tract. Semin Diagn Pathol 26: 77-88, 2009.
13. Huang DF and Chen WS: Images in clinical medicine. Lupus-associated intestinal vasculitis. N Engl J Med 361: e3, 2009.

14. Lee CK, Ahn MS, Lee EY, Shin JH, Cho YS, Ha HK, Yoo B and Moon HB: Acute abdominal pain in systemic lupus erythematosus: Focus on lupus enteritis (gastrointestinal vasculitis). Ann Rheum Dis 61: 547-550, 2002.

15. Lian TY, Edwards CJ, Chan SP and Chng HH: Reversible acute gastrointestinal syndrome associated with active systemic lupus erythematosus in patients admitted to hospital. Lupus 12: 612-616, 2003.

16. Passam FH, Diamantis ID, Perisinaki G, Saridaki Z, Kritikos H, Georgopoulos D and Boumpas DT: Intestinal ischemia as the first manifestation of vasculitis. Semin Arthritis Rheum 34: 431-441, 2004

17. Ju JH, Min JK, Jung CK, et al: Lupus mesenteric vasculitis can cause acute abdominal pain in patients with SLE. Nat Rev Rheumatol 5: 273-281, 2009.

18. Hsu CL, Chen KY, Yeh PS, et al: Outcome and prognostic factors in critically ill patients with systemic lupus erythematosus: A retrospective study. Crit Care 9: R177-R183, 2005.

19. Tan TC, Wansaicheon GK and Thong BY: Acute onset of systemic lupus erythematosus with extensive gastrointestinal and genitourinary involvement. Lupus 21: 1240-1243, 2012.

20. Majithia R, Joy G, Liang J and Olden K: Acute abdominal pain in systemic lupis erythematosus. Gastrointest Endosc 75: 1267-1268, 2012.

21. Lee J, Jung HS, Nam HC, Kwok SK, Ju JH, Park KS, Kim HY and Park SH: Fulminant amoebic colitis mimicking intestinal vasculitis in a patient with systemic lupus erythematosus. Lupus 21: 1351-1355, 2012.

22. Kishimoto M, Nasir A, Mor A and Belmont HM: Acute gastrointestinal distress syndrome in patients with systemic lupus erythematosus. Lupus 16: 137-141, 2007. 